\title{
MicroRNA-499 rs3746444 polymorphism in Egyptian children with biliary atresia
}

\author{
Eman Gawish', Elhamy Abd El-Monem¹, Mona El-Abd'1, Gihan Ahmed Sobhy², Heba Ghanem¹ \\ 'Department of Clinical Pathology, National Liver Institute - Menoufia University, Menoufia, Egypt \\ 2Department of Pediatric Hepatology, Gastroenterology and Nutrition, National Liver Institute - Menoufia University, Menoufia, Egypt
}

\begin{abstract}
Aim of the study: We aimed to evaluate the association of microRNA-499 rs3746444 polymorphism and biliary atresia (BA) risk and its correlation with clinic-pathologic features of BA.

Material and methods: This study was performed on 300 Egyptian children (100 BA cases, 100 cases with cholestatic liver diseases other than BA and 100 healthy controls). Routine laboratory investigations, clinical examination and abdominal ultrasound were done. All infants were genotyped for miR-499 single nucleotide polymorphisms (SNPs) (rs3746444 A>G) by real-time polymerase chain reaction (PCR) fluorescence detection on a Rotor Gene Real Time PCR System (QIAGEN, GmbH) using fluorescent labeled probes.

Results: The AG genotype was the most prevalent genotype of miR-499 rs3746444 among the studied groups. A significantly higher frequency of the rs3746444 $\mathrm{G}$ allele was found in the BA cases than the other groups (odds ratio $=1.62$ ). This polymorphism was also correlated with the degree of fibrosis in BA cases $(p<0.05)$. The miR-499 rs3746444 polymorphism (GG genotype) was significantly associated with severe form of BA and bad prognosis after the Kasai operation ( $p<0.05)$. miR-499 rs3746444 polymorphism had no effect on the clinic-pathological features or the liver function status in the non-BA group.

Conclusions: There is an association between the miR-499 SNP genotypes and the occurrence of BA. The variant allele $G$ is the predominant allele in the BA group and is associated with severe liver inflammation and bad prognosis after the Kasai operation.
\end{abstract}

Key words: biliary atresia, cholestatic liver diseases, Kasai operation, miR-499.

\section{Address for correspondence:}

Dr. Gihan Ahmed Sobhy, Department of Pediatric Hepatology, Gastroenterology and Nutrition, National Liver Institute Menoufia University, 32511 Shebin El-koom, Menoufia, Egypt, e-mail: GihanSohy2020@gmail.com

\section{Introduction}

Biliary atresia (BA) is an obliterative cholangiopathy characterized by inflammation of the bile ducts leading to progressive fibro-obliteration of the extrahepatic biliary tree and, to a variable extent, the intrahepatic bile ducts. The progressive destruction of the intra- and extrahepatic bile ducts leads to cholestasis, liver fibrosis and cirrhosis [1]. The exact etiology and pathogenesis of BA remain unknown. It is believed that genetic factors may have a significant role in BA pathogenesis [2].
The microRNAs (miRNAs) and their target genes cooperatively control life processes as they participate in complex regulatory networks and control gene expression in all biological processes, including cell development, immune responses, aging and cell death [3].

Many researchers have recently reported that the aberrant expression of miRNAs in hepatic tissue was related to the pathogenesis of liver disease, and different types of liver injury can elicit different miRNA secretion patterns and concentrations. Therefore, identifying disease-specific miRNAs could be used as potential diagnostic and therapeutic tools [4]. Single nucleotide polymorphisms 
(SNPs) are the most common genetic variations in a population [5]. These single-nucleotide polymorphisms may have a potential to affect the efficiency of miRNA binding to the target sites or can create or disrupt binding sites. The resulting gene dysregulation may involve changes in phenotype and may eventually prove critical for the susceptibility to different disease processes [6].

miR-499 participates in many biological processes which are crucial in the development and progression of many disease conditions [7]. An important polymorphism in the miR-499 with A to G change (rs3746444) was reported in 2008. This polymorphism is located in the stem region opposite to the mature miR- 499 sequence and results in a change in the stem structure of miR-499. Compared with the A allele, the secondary structure for the $\mathrm{G}$ allele is less stable [8].

The aim of this study was to evaluate the association of miRNA-499 rs3746444 A>G polymorphism with BA risk and its correlation with clinic-pathologic features of BA in Egyptian children.

\section{Material and methods}

This study was carried out at laboratory medicine department, National Liver Institute, Menoufia University, Egypt from October 2017 to November 2019. This study was conducted on 300 pediatric patients (100 BA patients, 100 non-BA patients, and 100 matched healthy controls). Among the 100 patients with BA 86 patients underwent Kasai portoenterostomy. The non-BA group included 64 cases with progressive familial intrahepatic cholestasis (PFIC), 9 cases with Alagille syndrome, 1 case with non-syndromic bile duct paucity, 2 cases with galactosemia, and 24 cases with idiopathic neonatal hepatitis. They were collected from the inpatient ward and outpatient clinic of pediatric hepatology, gastroenterology and nutrition department, National Liver Institute - Menoufia University, Egypt.

Diagnosis of BA was confirmed by intra-operative cholangiography when possible before corrective surgery (Kasai operation), and patients were subjected to intraoperative wedge liver biopsy. The diagnosis of BA was ruled out in patients with cholestasis due to causes other than BA. The diagnosis of these patients depended on a set of specific laboratory tests according to the expected etiology, liver imaging, liver biopsy, negative intra-operative cholangiography finding (in some patients), and applying the BA score [9]. The control group comprised 100 age- and gender-matched children without any evidence of liver disease. Ethical committee approval of the National Liver Institute was taken and informed written consent was obtained from all patients' and controls' legal health authorities before involvement in the study.
All individuals were subjected to clinical assessment, including full history and clinical examination, abdominal ultrasound, and routine laboratory investigations comprising liver function tests: total bilirubin, direct bilirubin, albumin, total protein, aspartate aminotransferase (AST), alanine aminotransferase (ALT) and $\gamma$-glutamyl transferase $(\gamma-\mathrm{GT})$ on the Cobas 6000 analyzer (c501 module), which is a photometric unit of the autoanalyzer, serum hepatitis $\mathrm{C}$ virus antibody (HCV-Abs) and serum hepatitis $\mathrm{B}$ virus surface antigen (HBsAg) by electrochemiluminescence immunoassay, complete blood count (CBC) using the Sysmex XT1800i automated hematology analyzer, international normalized ratio (INR) by Dade Behring Marburg GmbH, D-35041 Marburg, Germany, miRNA-499 rs3746444 gene polymorphism analysis by real-time polymerase chain reaction (PCR), and allelic discrimination analysis.

After the Kasai operation, those who underwent the Kasai operation were subjected to evaluation six months after the procedure by total bilirubin, direct bilirubin, serum albumin, and INR determination.

\section{MicroRNA-499 rs3746444 genotyping analysis}

Deoxyribonucleic acid (DNA) extraction: DNA was extracted from whole blood samples using the Thermo Scientific Gene JET Whole Blood Genomic DNA Purification Mini Kit, as recommended by the manufacturer.

TaqMan SNP Genotyping assay: MicroRNA-499 Rs3746444 was genotyped by Rotor Gene real-time PCR fluorescence (QIAGEN, GmbH) using fluorescent labeled probes which are sequence-specific oligonucleotides with a fluorophore and a quencher. Two TaqMan probes, each specific for one of the available alleles (A or G), are present in the reaction, specifically annealing to the target region between the two primers. The proximity of the fluorophore with the quencher results in efficient quenching of fluorescence from the fluorophore. The Taq DNA polymerase extends the primer. When the enzyme reaches the TaqMan probe, its 5'-3' exonuclease activity degrades the probe, resulting in physical separation of the fluorophore from the quencher. Increased fluorescence associated with the measured $\mathrm{Rn}$ values from the released fluorophore indicates which alleles are present in the sample.

PCR cycling conditions: 1 min cycle at $60^{\circ} \mathrm{C}$ (preread) and 90 min cycles (amplification steps) stage 1: hold at $95^{\circ} \mathrm{C}$ for $10 \mathrm{~min}$ and stage 2: 40 cycles (each cycle consists of $15 \mathrm{~s}$ at $95^{\circ} \mathrm{C}$ and $1 \mathrm{~min}$ at $60^{\circ} \mathrm{C}$ ) followed by 1 min cycle at $60^{\circ} \mathrm{C}$ for (post-read).

The SNP ID: Rs3746444 SNP in the MIR499 gene was determined by real-time detection PCR using the ABI TaqMan allelic discrimination kit (catalogue \#4351379, assay ID C-2142612-40, Applied Biosystems, 
Carlsbad, CA) and the Rotor Gene Real Time PCR System (QIAGEN, GmbH).

\section{Context sequence [VIC/FAM]}

ATGTTTAACTCCTCTCCACGTGAAC[A/G] TCACAGCAAGTCTGTGCTGCTTCCC (VIC dye for allele A, FAM dye for allele $G$ ).

\section{Statistical analysis}

Results were statistically analyzed using SPSS 18 (SPSS Inc., Chicago, IL). Quantitative data were shown as median and interquartile range (IQR). Qualitative data were expressed as frequency and percent. The $\chi^{2}$ test was used to measure the association between qualitative variables. The adjusted odds ratio (ORs) with $95 \%$ confidence interval (95\% CIs) was used. The Kruskal-Wallis test was done to compare medians of more than 2 sets of quantitative data and the Mann-Whitney $U$ test was done to compare medians of 2 sets of non-parametrically distributed quantitative data. The $p$ (probability) value was considered to be of statistical significance if it was less than 0.05 .

\section{Results}

All groups were gender and age matched (Table 1). The levels of liver profile tests (AST, ALT, $\gamma$-GT, total

Table 1. Gender, age and baseline laboratory investigations among the studied groups

\begin{tabular}{|c|c|c|c|c|}
\hline Variable & $\begin{array}{c}\text { Control } \\
(n=100)\end{array}$ & $\begin{array}{c}\text { Non-BA } \\
(n=100)\end{array}$ & $\begin{array}{c}\text { BA } \\
(n=100)\end{array}$ & $P$-value \\
\hline \multicolumn{4}{|l|}{ Gender $[n(\%)]$} & \multirow[t]{3}{*}{0.530} \\
\hline Male & $52(52 \%)$ & $56(56 \%)$ & $48(48 \%)$ & \\
\hline Female & $48(48 \%)$ & $44(44 \%)$ & $52(52 \%)$ & \\
\hline \multicolumn{4}{|l|}{ Age (months) } & \multirow[t]{3}{*}{0.764} \\
\hline Median (IQR) & $20.00(14.00)$ & $22.00(15.75)$ & $19.00(14.00)$ & \\
\hline Range (min-max) & $7.00-48.00$ & $8.00-50.00$ & $9.00-45.00$ & \\
\hline \multicolumn{4}{|l|}{$\operatorname{ALT}(\mathrm{U} / \mathrm{I})$} & \multirow{3}{*}{$\begin{array}{l}p 1<0.001 \\
p 2<0.001 \\
p 3=0.008\end{array}$} \\
\hline Median (IQR) & $16(6)$ & $116(165)$ & $164(125)$ & \\
\hline Range (min-max) & $10.00-30.00$ & $32.00-450.00$ & $42.00-425.00$ & \\
\hline \multicolumn{4}{|l|}{ AST (U/I) } & \multirow{3}{*}{$\begin{array}{l}p 1<0.001 \\
p 2<0.001 \\
p 3=0.005\end{array}$} \\
\hline Median (IQR) & $19.00(8.75)$ & $88(101.00)$ & $135(98)$ & \\
\hline Range (min-max) & $11.00-31.00$ & $18.00-299.00$ & $23.00-409.00$ & \\
\hline \multicolumn{4}{|l|}{$\gamma$-GT $(\mathrm{U} / \mathrm{I})$} & \multirow{3}{*}{$\begin{array}{l}p 1<0.001 \\
p 2<0.001 \\
p 3<0.001\end{array}$} \\
\hline Median (IQR) & $17.00(8.0)$ & $73.00(102.00)$ & $551(319.00)$ & \\
\hline Range (min-max) & $10.00-27.00$ & $20.00-240.00$ & $140.00-1200.00$ & \\
\hline \multicolumn{4}{|l|}{ Total bilirubin (mg/dl) } & \multirow{3}{*}{$\begin{array}{l}p 1<0.001 \\
p 2<0.001 \\
p 3<0.001\end{array}$} \\
\hline Median (IQR) & $0.7(0.2)$ & $5.38(6.92)$ & $8.8(3.88)$ & \\
\hline Range (min-max) & 0.4-0.97 & $0.3-18$ & $2.3-20$ & \\
\hline \multicolumn{4}{|l|}{ Direct bilirubin (mg/dl) } & \multirow{2}{*}{$\begin{array}{l}p 1<0.001 \\
p 2<0.001\end{array}$} \\
\hline Median (IQR) & $0.15(0.12)$ & $4.0(5.0)$ & $6.50(3.58)$ & \\
\hline \multicolumn{4}{|l|}{ Albumin $(g / d l)$} & \multirow{3}{*}{$\begin{array}{l}p 1<0.001 \\
p 2<0.001 \\
p 3=0.012\end{array}$} \\
\hline Median (IQR) & $4.6(0.58)$ & $4.0(0.90)$ & $3.90(1.00)$ & \\
\hline Range (min-max) & $3.70-5.20$ & $3.00-5.10$ & $2.30-5.20$ & \\
\hline \multicolumn{4}{|l|}{ INR value } & \multirow{3}{*}{$\begin{array}{l}p 1<0.001 \\
p 2<0.001 \\
p 3<0.001\end{array}$} \\
\hline Median (IQR) & $1.02(0.06)$ & $1.10(0.11)$ & $1.2(0.18)$ & \\
\hline Range (min-max) & $0.90-1.10$ & $0.90-1.6$ & $0.96-1.9$ & \\
\hline \multicolumn{4}{|l|}{$\mathrm{HB}(\mathrm{g} / \mathrm{dl})$} & \multirow{3}{*}{$\begin{array}{l}p 1<0.001 \\
p 2<0.001 \\
p 3=0.111\end{array}$} \\
\hline Median (IQR) & $13.15(1.88)$ & $10.3(1.9)$ & $9.65(2.1)$ & \\
\hline Range (min-max) & $11.1-15$ & $6.5-14.7$ & $7.4-12.0$ & \\
\hline
\end{tabular}

p1 - significance between the control and non-BA groups, $p 2$ - significance between the control and $B A$ groups, $p 3$ - significance between the non-BA and $B A$ groups $B A$ - biliary atresia, ALT - alanine aminotransferase, AST - aspartate aminotransferase, $\gamma$-GT - gamma-glutamyl transferase, INR - international normalized ratio, HB - hemoglobin 
Table 2. Genotype distribution and allele frequencies of miR-499 polymorphism (rs3746444) among the studied groups

\begin{tabular}{|c|c|c|c|c|}
\hline $\begin{array}{l}\text { Polymorphism of miR-499 } \\
\text { (rs3746444) }\end{array}$ & $\begin{array}{l}\text { Control } \\
(n=100)\end{array}$ & $\begin{array}{l}\text { Non-BA } \\
(n=100)\end{array}$ & $\begin{array}{c}\text { BA } \\
(n=100)\end{array}$ & $P$-value \\
\hline \multicolumn{4}{|l|}{ Genotypes [n (\%)] } & 0.019 \\
\hline $\mathrm{AA}$ & $42(42 \%)$ & $41(41 \%)$ & 31 (31\%) & \\
\hline$A G$ & $50(50 \%)$ & $49(49 \%)$ & $46(46 \%)$ & \\
\hline GG & $8(8 \%)$ & $10(10 \%)$ & $23(23 \%)$ & \\
\hline \multicolumn{4}{|l|}{ Dominant model } & 0.208 \\
\hline $\mathrm{AA}$ & $42(42 \%)$ & $41(41 \%)$ & 31 (31\%) & \\
\hline$A G+G G$ & $58(58 \%)$ & $59(59 \%)$ & $69(69 \%)$ & \\
\hline \multicolumn{4}{|l|}{ Recessive model } & 0.004 \\
\hline$A A+A G$ & $92(92 \%)$ & $90(90 \%)$ & $77(77 \%)$ & \\
\hline GG & $8(8 \%)$ & $10(10 \%)$ & $23(23 \%)$ & \\
\hline \multicolumn{4}{|l|}{ Alleles [n(\%)] } & 0.014 \\
\hline A & $134(67 \%)$ & $131(65.5 \%)$ & 108 (54\%) & \\
\hline$G$ & $66(33 \%)$ & 69 (34.5\%) & $92(46 \%)$ & \\
\hline
\end{tabular}

BA - biliary atresia

bilirubin, direct bilirubin, albumin, and INR) and hemoglobin values showed statistically significant differences $(p<0.05)$ among the three studied groups, except for hemoglobin; no significant difference was found between BA and non-BA group (Table 1).

Our results revealed a significant difference concerning the genotype distribution among the three studied groups $(p<0.05)$. In the dominant model (GG + AG vs. AA), the distribution among the studied groups revealed no significant difference $(p>0.05)$, while in the recessive model (GG vs. AA $+A G$ ) a significant difference was found between the studied groups $(p<0.05)$. The wild allele A was more frequent in the control and non-BA groups while the variant allele $\mathrm{G}$ was more frequent in the BA group $(p<0.05)$ (Table 2 ).

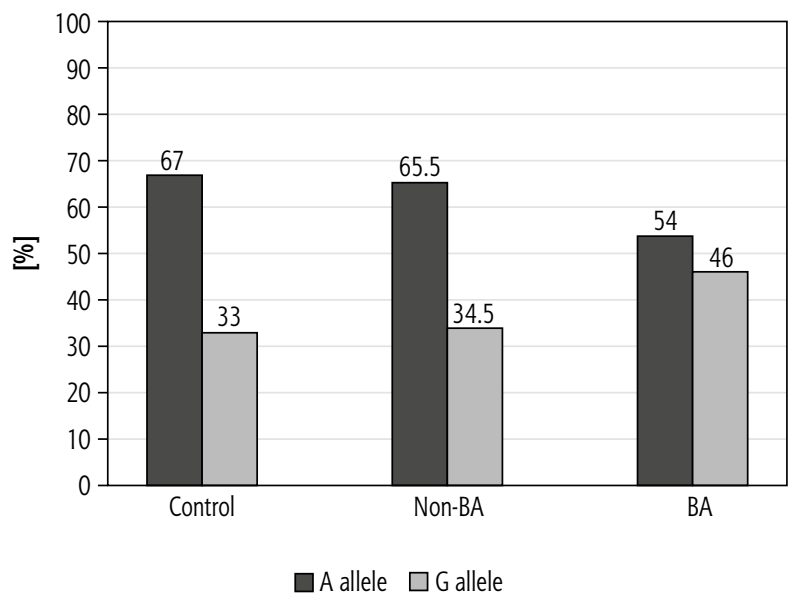

Fig. 1. Allele frequency of miR-499 SNP (rs3746444) in the three studied groups
The comparison of genotype distribution and allele frequencies of miR-499 SNP (rs3746444) between control and BA groups showed that GG genotype was associated with a significantly increased risk of BA as compared with AA genotype (OR = 3.9, 95\% CI: 1.54 9.86, and $p=0.003$ ) while AG genotype was not significantly associated with the risk of BA as compared with AA genotype $(\mathrm{OR}=1.25,95 \% \mathrm{CI}$ : $0.68-2.3$, and $p=0.481)$.

The subjects with the variant $G$ allele had a significant risk of BA compared with wild allele A (Fig. 1) $(\mathrm{OR}=1.73,95 \% \mathrm{CI}: 1.15-2.59$ and $p=0.008)$. The $\mathrm{G}$ allele also showed a significant association with BA risk in the recessive model [GG vs. $\mathrm{AG}+\mathrm{AA}(\mathrm{OR}=3.44$, 95\% CI: $1.45-8.11$, and $p=0.003$ )], while the dominant model showed no significant association with disease risk $(p=0.106)$.

The comparison of genotype distribution and allele frequencies of miR-499 SNP (rs3746444) between BA and non-BA groups showed that GG genotypes were associated with a significantly increased risk of BA as compared with AA genotype (OR = 3.04, 95\% CI: 1.27 7.31 , and $p=0.011$, while AG genotypes were not significantly associated with the risk of BA as compared with AA genotype (OR $=1.24,95 \% \mathrm{CI}$ : 0.67-2.30, and $p=0.491)$. The subjects with the variant $G$ allele had a highly significant risk of BA compared with wild allele A (OR $=1.62,95 \% \mathrm{CI}: 1.08-2.42$, and $p=0.019)$. The $G$ allele also showed a significant association with risk of BA in the recessive model [GG vs. AG + AA $(\mathrm{OR}=2.69,95 \% \mathrm{CI}: 1.21-6.00$, and $p=0.013)$, while in the dominant model no statistical significance was 
Table 3. Comparison between different miR-499 genotypes regarding laboratory investigations, clinical data and histopathological assessment in the BA group

\begin{tabular}{|c|c|c|c|c|}
\hline \multirow[t]{2}{*}{ Parameters } & \multicolumn{3}{|c|}{ SNP miR-499 in BA group } & \multirow[t]{2}{*}{ Pairwise comparisons } \\
\hline & $\mathrm{AA}(n=31)$ & $\mathrm{AG}(n=46)$ & $\mathrm{GG}(n=23)$ & \\
\hline \multicolumn{4}{|l|}{ Age (months) } & \multirow[t]{3}{*}{0.427} \\
\hline Median (IQR) & $17(16)$ & $20(10.75)$ & $17(11)$ & \\
\hline Range (min-max) & $9-45$ & $9-43$ & $9-44$ & \\
\hline \multicolumn{4}{|l|}{ Basal ALT (U/I) } & \multirow{3}{*}{$\begin{array}{l}p 1=1.000 \\
p 2<0.001 \\
p 3<0.001\end{array}$} \\
\hline Median (IQR) & $150(74)$ & $148(130)$ & $283(79)$ & \\
\hline Range (min-max) & $42-380$ & $42-368$ & $144-425$ & \\
\hline \multicolumn{4}{|l|}{ Basal AST (U/I) } & \multirow{3}{*}{$\begin{array}{l}p 1=1.000 \\
p 2=0.004 \\
p 3=0.001\end{array}$} \\
\hline Median (IQR) & $133(80)$ & $122(74.75)$ & $213(117)$ & \\
\hline Range (min-max) & $23-267$ & $23-409$ & $109-409$ & \\
\hline \multicolumn{4}{|l|}{ Basal $\gamma$-GT (U/I) } & \multirow{3}{*}{$\begin{array}{l}p 1=0.241 \\
p 2=0.001 \\
p 3=0.027\end{array}$} \\
\hline Mean $\pm S D$ & $495.21 \pm 220$ & $585.10 \pm 220$ & $744 \pm 291$ & \\
\hline Range (min-max) & $140-1000$ & $140-1152$ & $270-1200$ & \\
\hline \multicolumn{4}{|l|}{ Basal total bilirubin (mg/dl) } & \multirow{3}{*}{$\begin{array}{l}p 1=0.506 \\
p 2=0.004 \\
p 3=0.089\end{array}$} \\
\hline Median (IQR) & $8(4)$ & $8.68(4.88)$ & $10.3(5.2)$ & \\
\hline Range (min-max) & $2.3-14$ & $2.3-19$ & $4-20$ & \\
\hline \multicolumn{4}{|l|}{ Basal direct bilirubin (mg/dl) } & \multirow[t]{3}{*}{0.051} \\
\hline Mean \pm SD & $6.16 \pm 2.24$ & $6.81 \pm 2.92$ & $8.06 \pm 3.22$ & \\
\hline Range (min-max) & $1.3-12$ & 1.30-15.6 & $2.00-15.6$ & \\
\hline \multicolumn{4}{|l|}{ Basal ALB (U/I) } & \multirow[t]{3}{*}{0.074} \\
\hline Mean \pm SD & $3.9 \pm 0.75$ & $3.78 \pm 0.7$ & $3.45 \pm 0.73$ & \\
\hline Range (min-max) & $2.45-5.2$ & $2.4-5.2$ & 2.3-5.1 & \\
\hline \multicolumn{4}{|l|}{ Basal INR } & \multirow[t]{3}{*}{0.136} \\
\hline Median (IQR) & $1.2(0.08)$ & $1.2(0.16)$ & $1.2(0.3)$ & \\
\hline Range (min-max) & $1-1.8$ & $0.96-1.7$ & $0.96-1.9$ & \\
\hline \multicolumn{4}{|l|}{ Basal hemoglobin (g/dl) } & \multirow[t]{3}{*}{0.132} \\
\hline Median (IQR) & $10.5(1.8)$ & $9.60(2.35)$ & $9.40(1.3)$ & \\
\hline Range (min-max) & $7.4-12$ & $7.5-12$ & 7.4-11 & \\
\hline Ascites & $26(83.9)$ & $42(91.3)$ & $16(69.6)$ & 0.074 \\
\hline Splenomegaly & $15(48.4)$ & $14(30.4)$ & $7(30.4)$ & 0.224 \\
\hline Hepatomegaly & $6(19.4)$ & $18(39.1)$ & $8(34.8)$ & 0.180 \\
\hline \multicolumn{4}{|l|}{ Degree of fibrosis } & \multirow[t]{4}{*}{0.021} \\
\hline Mild fibrosis & $10(32.3 \%)$ & $16(34.8 \%)$ & $2(8.7 \%)$ & \\
\hline Moderate fibrosis & $13(41.9 \%)$ & $20(43.5 \%)$ & $8(34.8 \%)$ & \\
\hline Severe fibrosis & $8(25.8 \%)$ & $10(21.7 \%)$ & $13(56.5 \%)$ & \\
\hline
\end{tabular}

p1 - significance between the AA and AG genotypes, $p 2$ - significance between the AA and GG genotypes, $p 3$ - significance between the AG and GG genotypes ALT - alanine aminotransferase, AST - aspartate aminotransferase, $\gamma$-GT - $\gamma$-glutamyl transferase, INR - international normalized ratio

found $[\mathrm{AA}$ vs. $\mathrm{AG}+\mathrm{GG}(\mathrm{OR}=1.55,95 \% \mathrm{CI}: 0.86-$ 2.77, and $p=0.141)]$.

Concerning the laboratory, clinical and histopathological assessment, there was a significant difference between different genotypes in the BA group regarding baseline ALT, AST, $\gamma-\mathrm{GT}$, and total bilirubin.
Furthermore, the multiple pairwise comparisons regarding the levels of ALT, AST and $\gamma$-GT showed that no significant difference was found between AA and AG genotypes, while a significant difference was found between the AA and GG genotypes and AG and GG genotypes $(p<0.05)$ with higher values with GG geno- 
Table 4. Changes in the laboratory parameters before and after the Kasai operation among genotypes of miR-499 SNP (rs3746444) polymorphism in the biliary atresia group

\begin{tabular}{|c|c|c|c|c|}
\hline \multirow{2}{*}{$\begin{array}{l}\text { Change in lab parameters } \\
\text { (after - before) }\end{array}$} & \multicolumn{3}{|c|}{ Genotypes of miR-499 gene polymorphism in BA group } & \multirow[t]{2}{*}{$P$-value } \\
\hline & $\begin{array}{c}\text { AA } \\
(n=29)\end{array}$ & $\begin{array}{c}\text { AG } \\
(n=42)\end{array}$ & $\begin{array}{c}\text { GG } \\
(n=15)\end{array}$ & \\
\hline \multicolumn{4}{|l|}{ Total bilirubin (mg/dl) } & \multirow{3}{*}{$\begin{array}{l}p 1=0.748 \\
p 2=0.011 \\
p 3=0.092\end{array}$} \\
\hline Median (IQR) & $-4.3(2.88)$ & $-3.80(5.16)$ & $1.5(8.3)$ & \\
\hline Range (min-max) & $-7.6-2.00$ & $-13.00-4.00$ & $-7.30-4.2$ & \\
\hline \multicolumn{4}{|l|}{ Direct bilirubin (mg/dl) } & \multirow{3}{*}{$\begin{array}{l}p 1=0.922 \\
p 2=0.011 \\
p 3=0.072\end{array}$} \\
\hline Median (IQR) & $-3.6(2.35)$ & $-3.15(4.03)$ & $1.00(5.70)$ & \\
\hline Range (min-max) & $-6.3-1.6$ & $-8.60-2.90$ & $-5.60-3.30$ & \\
\hline \multicolumn{4}{|l|}{ Albumin (g/dl) } & \multirow[t]{3}{*}{0.090} \\
\hline Median (IQR) & $0.22(0.37)$ & $0.12(0.33)$ & $-0.05(0.52)$ & \\
\hline Range (min-max) & $-0.50-1.00$ & $-0.50-1.00$ & $-1.00-1.00$ & \\
\hline \multicolumn{4}{|l|}{ INR value } & \multirow{3}{*}{$\begin{array}{l}p 1=0.054 \\
p 2=0.001 \\
p 3=0.158\end{array}$} \\
\hline Median (IQR) & $-0.1(0.10)$ & $-0.10(0.25)$ & $0.20(0.40)$ & \\
\hline Range (min-max) & $-0.4-0.10$ & $-0.30-0.30$ & $-0.30-0.60$ & \\
\hline
\end{tabular}

type. Regarding total bilirubin, a significant difference was found between AA and GG genotypes, while no significant difference was found between AA and AG genotypes and AG and GG genotypes. No significant difference was found between different genotypes regarding albumin, INR and hemoglobin values. The present study showed no significant difference in the clinical data (hepatomegaly and splenomegaly) among different genotypes in the BA group, while there was a significant difference among different genotypes as regards the degree of fibrosis (Table 3 ).

Table 4 shows a significant difference $(p<0.05)$ regarding change in the laboratory parameters before and after the Kasai operation among genotypes of miR-499 SNP (rs3746444) polymorphism in the BA group except for albumin; no significant difference was found $(p>0.05)$. Furthermore, the multiple pairwise comparison showed no significant difference in $\mathrm{TB}, \mathrm{DB}$ and INR between $\mathrm{AA}$ and $\mathrm{AG}$ genotypes. Also, no significant difference was found between AG and GG genotypes, while a significant difference was found between AA and GG genotypes regarding TB, $\mathrm{DB}$ and INR.

\section{Discussion}

Biliary atresia is the most common cause of end stage liver disease in infants and is associated with poor prognosis and high mortality. The etiology of BA is not known, but irrespective of the cause, histological evidence at diagnosis supports the involvement of the immune system in mediating injury to the bile duct epithelium, which is facilitated by adhesion molecules that help recruitment of inflammatory cells [10].

In this study, we investigated the effects of the miR-499 rs3746444 polymorphism on the risk of BA, the association with the liver function status and the clinic-pathologic data and whether this gene polymorphisms affect the prognosis after the Kasai operation in patients who underwent this procedure. To the best of our knowledge, this is the first study to investigate miR-499 rs3746444 gene polymorphism in cases of BA among the Egyptian population.

The AG genotype was the most prevalent genotype in the studied population, while the GG genotype was associated with a significantly increased risk of BA by 2.9 times as compared to AA and AG. The subjects with the variant $G$ allele had a significant risk of BA as compared with wild allele A. Additionally, in the recessive model, the $G$ allele showed a significant association with increased risk of BA by 2.4 times. On the other hand, the distribution of miR-499 SNP (rs3746444) genotypes, alleles frequencies, dominant, or recessive models were non-significantly associated with the risk of non-BA disease. Consistent with our study, Fattah et al. investigated the association between miR-499 SNP (rs3746444) and rheumatoid arthritis (RA) [11]. They demonstrated that the AG genotype was the most prevalent in the study population. In addition, it was found that the $\mathrm{G}$ allele was associated with RA. Several lines of evidence suggest that miR-499a plays critical roles in orchestrating the immune response in various human disorders. Many studies have claimed a key role for miR-499 in modulating the immune response, cell 
proliferation, apoptosis, inflammation, and immune response. Exploring gene targets of miR-499a identified inflammatory-related gene targets, including interleukin (IL)-13 and IL-23, IL6, IL-2 and IL-17 [12].

The significantly higher values of the liver function tests with GG genotype at presentation and its association with the severity of fibrosis indicate that there is an effect of the gene polymorphism on the disease severity due to its effect on the biological processes such as inflammation and immune response. In agreement with our results, Shan et al. observed that GG genotype was significantly associated with severity of fibrosis in BA patients, but they failed to observe any difference in the preoperational liver function indices [13].

Regarding the effect of genotypes on the outcome 6 months after the Kasai operation, it was found that the change in total and direct bilirubin, and INR levels were significantly more improved in patients carrying AA or AG genotypes but not GG, revealing bad prognosis after the Kasai operation for patients with the GG genotype.

Finally, the obtained data support our assumption that mutation in the seeding area of miR-499 may affect affinity of miRNA, interfering with the perfect binding to target mRNA, and thus may modify BA susceptibility and affect the concentration of inflammatory cytokines, which may be associated with disease severity and outcome after the Kasai operation. So, polymorphism of miR-499 may not only play a role in biliary pathogenesis but also have a longterm effect on the post-Kasai prognosis.

\section{Conclusions}

There is an association between the miR-499 SNP genotypes and the incidence of BA in Egyptian patients. The variant allele $\mathrm{G}$ was higher in the BA group than the other two groups. miR-499 rs3746444 polymorphism was significantly associated with severe liver inflammation and bad prognosis after the Kasai operation.

\section{Acknowledgements}

We would like to thank all our professors and colleagues at the Department of Clinical Pathology and Department of Pediatric Hepatology, National Liver Institute - Menoufia University for their valuable assistance to accomplish this work.

\section{Disclosure}

The authors declare no conflict of interest.

\section{References}

1. Lillegard JB, Miller AC, Flake AW. Biliary atresia. In: Fundamentals of pediatric surgery. $2^{\text {nd }}$ ed. Springer International Publishing CY, Cham 2017; 629-636.

2. Pei J, Dong R, Yang Y, et al. Association of STAT4 genetic polymorphisms with biliary atresia in Chinese patients. Int J Clin Exp Pathol 2017; 10: 3451-3455.

3. Murakami Y, Kawada N. MicroRNAs in hepatic pathophysiology. Hepatol Res 2017; 47: 60-69.

4. Kennedy L, Francis H, Meng F, et al. Diagnostic and therapeutic potentials of microRNAs in cholangiopathies. Liver Res 2017; 1: 34-41.

5. Wang Z, Wu J, Zhang G, et al. Associations of miR-499 and miR-34b/c polymorphisms with susceptibility to hepatocellular carcinoma: an evidence-based evaluation. Gastroenterol Res Pract 2013; 2013: 719202.

6. Chen C, Yang S, Chaugai S, et al. Meta-analysis of Hsa-mir-499 polymorphism (rs3746444) for cancer risk: evidence from 31 case-control studies. BMC Med Genet 2014; 15: 126.

7. Fan C, Chen C, Wu D. The association between common genetic variant of microRNA-499 and cancer susceptibility: a meta-analysis. Mol Biol Rep 2013; 40: 3389-3394.

8. Hayashida M, Matsuura T, Kinoshita Y, et al. Parameters that help to differentiate biliary atresia from other diseases. Pediatr Int 2017; 59: 1261-1265.

9. El-Guindi MA, Sira MM, Sira AM, et al. Design and validation of a diagnostic score for biliary atresia. J Hepatol 2014; 61: 116-123.

10. Kilgore A, Mack C. Update on investigations pertaining to the pathogenesis of biliary atresia. Pediatr Surg Int 2017; 33: 1233-1241.

11. Fattah S, Ghattas M, Saleh S, et al. Pre-micro RNA-499 gene polymorphism rs3746444 T/C is associated with susceptibility to rheumatoid arthritis in Egyptian population. Indian J Clin Biochem 2017; 33: 96-101.

12. Song J, Shao H. SNP array in hematopoietic neoplasms: a review. Microarrays 2015; 5: 100-103.

13. Shan Y, Shen N, Han L, et al. MicroRNA-499 Rs3746444 polymorphism and biliary atresia. Dig Liver Dis 2016; 48: 423-428. 